\title{
Anesthetic Strategy for a Femur Fracture Surgery in a Patient with Hemophilia B (Factor IX Deficiency): A Case Report
}

\author{
Thiago MS
}

Department of Medicine, University of Southern Santa Catarina - UNISUL. Tubarão Brazil

*Corresponding author: Thiago MS, Department of Medicine, University of Southern Santa Catarina - UNISUL. Tubarão Brazil, Tel: +55(48)3622-1442, E-mail: thiagosakae@gmail.com

Citation: Thiago MS (2017) Anesthetic Strategy for a Femur Fracture Surgery in a Patient with Hemophilia B (Factor IX Deficiency): A Case Report. SAJ Case Rep 4: 205

Article history: Received: 16 February 2017, Accepted: 17 March 2017, Published: 19 March 2017

\section{Abstract}

Background: Hemophilia is a hemorrhagic trend affects mostly males (X-related recessive disease). In $85 \%$ of cases it is caused by factor VIII deficiency, being called hemophilia A or classic hemophilia. In approximately $15 \%$ of cases there is factor IX deficiency (hemophilia B).

Purpose: The objective of this report was to describe the case of a patient with Hemophilia B underwent a femur fracture surgery.

Case report: Male patient-18 years old, $81.7 \mathrm{~kg}, 183 \mathrm{~cm}$, carrying hemophilia B, victim of a motorcycle traffic accident, was admitted in emergency room with Glasgow 15, excoriations in arms and thorax, and a femur fracture. He was first subjected to external fixation and posteriorly underwent surgery to correct a distal femur fracture.

Discussion: Hemophiliac patients' administration has made strides. Hemophilia ought not to be a contraindication for an obtrusive strategy; be that as it may, various conditions are required to give effective surgery and an uncomplicated and safe postoperative course.

Keywords: Hemophilia; Case Report; Anesthesiology; Coexistent Diseases; Monitoring; Intraoperative; Intraoperative Period

\section{Introduction}

Hemophilia is a hemorrhagic trend affects mostly males (X-related recessive disease). In $85 \%$ of cases it is caused by factor VIII deficiency, being called hemophilia A or classic hemophilia. In approximately $15 \%$ of cases there is factor IX deficiency (hemophilia B) $[1-3]$.

Hemophilia B is characterized into severe (factor IX $<1 \%)$, moderate $(1 \%-5 \%)$, or mild (5\%-40\%) phenotypes in light of the plasma component IX action of influenced people [4]. A variety of of underlying mutations have been identified and linked with different levels of clinical severity. Severe or moderate hemophilia B is considered as $<5 \mathrm{IU} / \mathrm{dL}$ factor IX activity $[4,5]$. The serious phenotype is portrayed by unconstrained and repetitive draining scenes into joints and muscles, with hemarthroses being the dominating reason for long haul disability [5]. The moderate phenotype is described by incidental unconstrained drains and delayed seeping with minor injury or surgery. At last, patients with the gentle phenotype once in a while exhibit unconstrained draining yet may have critical seeping with significant injury or surgery. Forceful component substitution is required fundamentally for patients with moderate and serious hemophilia B phenotypes [1-4].

Hemophilia ought not to be a contraindication for an invasive procedure; be that as it may, various conditions are required to give fruitful surgery and an uncomplicated and safe postoperative course [6].

In any capacity, when these patients are submitted to surgical procedures, it merits uncommon consideration and a multidisciplinary group of wellbeing experts educated about the sickness, including qualified hematologist, surgeon and anesthesiologist [3,7,8]. The objective of this report was to describe the case of a patient with Hemophilia B underwent a femur fracture surgery.

\section{Case Report}

Male patient, 18 years old, weight 81.7 kilograms, $183 \mathrm{~cm}$ in height, carrier hemophilia B (factor IX deficiency). He was a victim of a motorcycle traffic accident, was admitted in emergency room with Glasgow 15, normal cardiac and pulmonary auscultation, excoriations in arms and thorax, and a femur fracture. Blood pressure of $157 \mathrm{x} 83 \mathrm{mmHg}$ and heart rate of $126 \mathrm{bpm}$. The first 
surgery was conducted with general anesthesia to put an external orthopedic fixator. After damage controls, he had an elective surgery purpose to correct a distal femur fracture.

During pre-anesthetic evaluation, presented in good condition, hydrated, no fever and eupneic. Normal psychomotor development and no other comorbidities. Denied allergies, addictions and family and personal history negative for anesthetic complications. Evaluation Mallampati class me with good mouth opening and class II according to American Society of Anesthesiologists (ASA). Normal cardiac and pulmonary auscultation. Initial tests in emergency room showed hematocrit of $42.1 \%$, hemoglobin of 14.2 g.dL ${ }^{-1}$, leukocytes of $14.900 / \mathrm{mm}^{3}, 274.000 / \mathrm{mm}^{3}$ platelets, serum sodium of $147.1 \mathrm{mEq} . \mathrm{L}^{-1}$, serum potassium of $3.7 \mathrm{mEq} . \mathrm{L}^{-1}$, fasting blood glucose of $96 \mathrm{mg} . \mathrm{dL}^{-1}$, creatinine of $0.61 \mathrm{mg} \cdot \mathrm{dL}^{-1}$, urea of $16 \mathrm{mg} \cdot \mathrm{dL}^{-1}$, activation time prothrombin of 100.0\% (INR: 1,00), coagulation time 8 minutes, bleeding time 2 minutes 30 seconds and normal electrocardiogram.

On the third day of hospitalization factor IX was administered in a dose of 50 UI.kg-1 IV (4000UI) intravenous until fifth day. On the sixth day it was administered 7500UI (93.75 UI.kg-1) three hours before the surgery (Table 1).

\begin{tabular}{|c|c|c|c|c|c|}
\hline Day & $\begin{array}{c}\text { Hematocrit } \\
\%\end{array}$ & $\begin{array}{c}\text { Hemoglobin } \\
\mathbf{g . d L}^{-\mathbf{1}}\end{array}$ & $\begin{array}{c}\text { Platelets / } \\
\mathbf{m m}^{\mathbf{3}}\end{array}$ & $\begin{array}{c}\text { PT } \\
\text { (seconds) }\end{array}$ & $\begin{array}{c}\text { aPTT } \\
\text { (seconds) }\end{array}$ \\
\hline Admisson & 42.0 & 14.2 & 274000 & 12 & 32 \\
\hline Third & 40.2 & 13.9 & 271000 & 18 & 79 \\
\hline Fourth & 41.1 & 13.9 & 266000 & 14 & 39.5 \\
\hline Fifth & 41.1 & 13.6 & 258000 & 13 & 37.4 \\
\hline Sixth & 42.1 & 13.8 & 256000 & 13 & 37.1 \\
\hline \multicolumn{7}{|}{$\begin{array}{l}\text { PT- prothrombin time } \\
\text { aPTT - Activated Partial Thromboplastin Time }\end{array}$} \\
Table 1: Laboratory tests until in hospital days
\end{tabular}

The patient was placed supine, monitored, and pre medicated with clonidine 75 mcg intravenous (IV). At this moment tranexamic acid 8,0g (97.9 mg. $\left.\mathrm{kg}^{-1}\right)$ was administered preemptively. After pre-oxygenation, anesthesia was induced with fentanyl $350 \mu \mathrm{g}$, IV propofol $150 \mathrm{mg}$ IV and atracurium $40 \mathrm{mg}$ IV. The others adjuvants administered were cefazolin $2 \mathrm{~g}$, dexametasone $10 \mathrm{mg}$, ondansetron $8 \mathrm{mg}$, haloperidol $5 \mathrm{mg}$, morphine $8 \mathrm{mg}$, dipyrone $2 \mathrm{~g}$ and lidocaine $100 \mathrm{mg}$. There was no bradycardia events and low blood pressure were corrected with metaraminol 0,5 mg IV as necessary. Intubation was successful as Cormack-Lehane grade II without difficult. Anesthesia was maintained with sevoflurane and atracurium as an option for muscle relaxation. The monitoring was maintained sinus rhythm, saturation between $98 \%$ and $100 \%$, end tidal $\mathrm{CO}_{2}$ between 32 and 38 and mean arterial pressure between 73 and 90 throughout the period.

Diuresis total was $150 \mathrm{ml}\left(0.93 \mathrm{ml} \cdot \mathrm{kg}^{-1} \cdot \mathrm{h}^{-1}\right)$. The intraoperative blood gases and $\mathrm{pH}$ showed immediate postoperative between 7.34 and 7.38; $\mathrm{PO}_{2}$ between 98 and 121, bicarbonate between 22.6 and 26.7 and base excess between -3.2 and +2.5 . None packed red blood cells and bags of fresh plasma were necessary.

Total anesthesia time was 2 hours and 45 minutes and course proceeded with two episodes of intraoperative hypotension promptly treated with metaraminol $0,5 \mathrm{mg}$ in bolus. The patient was extubated taken at surgery and sent to the Intensive Care Unit. Postsurgical bleeding was not significant and the patient had discharge from hospital in three days without major complications.

\section{Discussion}

So far, there are few cases [9] of anesthetic procedures with hemophilia B published in the literature submitted to general anesthesia, since previous studies have described the management of hemophilia A and C $[3,6,7,10]$. Surgical treatment is a safe and reliable choice for addressing complications including hemophilia-related osteoarthropathy given the implementation of effective measures for treatment during the perioperative period [7]. The single most important factor which contributes to the severity of hemophilia is the nature of mutations. Even the phenotypically severe hemophilia cases can further be subclassified based on the type of mutations they carry. Even both hemophilia A and B mutation databases show that less severe gene defects are more common in hemophilia B than in A [11]. About hemophilia A it is severe if coagulation factor VIII level is below 1\%; it is moderate if it is between $1 \%$ and $5 \%$; and mild if it is above $5 \%[1,3,5]$ Normal factor VIII plasma levels vary $0.5 \mathrm{U} / \mathrm{mL}$ to $1.5 \mathrm{U} / \mathrm{mL}$ and each U/mL corresponds to $100 \%$ factor VIII found in $1 \mathrm{~mL}$ of plasma. Laboratory diagnosis of hemophilia A is based on prolonged TTPa and factor VIII deficiency, normal factor IX and von Willebrand factor levels [1,3].

The dose of recombinant coagulation factor IX was adjusted to sustain factor IX activity above $80 \%$, as suggested by Makino [9]. The patient showed a favorable course without hemorrhagic tendency. We could safely manage anesthesia without requiring allogeneic blood transfusion. The goal in this stage is to correct factor IX deficiency before the procedure, which is hematologist's responsibility. It must be known that patients need $100 \%$ correction of their factor IX before any surgical procedure, and this must be confirmed before surgery $[3,8,10]$. In Hemophilia A, checking ought to be accomplished by dosing variable VIII levels here and there a day. To the Hemophilia B, we can think similarly. In spite of the fact that not perfect, it is additionally conceivable to observing with serial TTPa estimation if serial element VIII or IX estimation is not accessible. Patients' coagulation will be viewed 
as satisfactory when the proportion between patients' TTPa and typical TTPa is equivalent to or underneath 1.2 [8].

Fresh frozen plasma is utilized subsequent to the 1950s to treat hemophilia. It contains 175 to $250 \mathrm{~mL}$ of volume for each sack with 70 to $90 \mathrm{U} . d L^{-1}$ variable VIII, component IX, von Willebrand element and other coagulating elements. It can supplant $15 \%$ to $20 \%$ component VIII with a volume of 800 to $1000 \mathrm{~mL}$. It might be utilized to treat known coagulation element insufficiencies in situations when the particular concentrate is not accessible. Prothrombin concentrate complex is comprised of prothrombin concentrate, elements IX and X and variable element VIII sums. Utilized as a part of hemophilia A in patients with component VIII inhibitors, in the measurements of 75 to $100 \mathrm{U.kg}^{-1}$. It is related to thromboembolic issues. Cryoprecipitate and Factor VIII concentrate are demonstrated to hemophilia A $[3,12,13]$. Desmopressin increases the level of Factor VIII and Factor von Willebrand. It is therefore useful in Hemophilia patients with mild to moderate (not useful in severe) [3,13]. Not affect the level of factor IX, which is contraindicated in hemophilia B [13]. The level of plasma IX factor increases approximately $1 \%$ per unit / $\mathrm{kg}$ of Factor IX infused. The recombinant factor IX is less replacement ( 0.8 adults and $0.7 \%$ in children). The half life of factor IX is 18 -24hs $[13,14]$.

Épsilon aminocaproic acid has a limited use because of low power and high toxicity [13]. Tranexamic acid is an adjuvant, mainly in factor XI deficient patients. Its use is indicated in minor bleeding of mucous during surgical and dental procedures. Use is contraindicated in patients with hematuria, severe chest surgery, or in patients haemophilia inhibitors (neutralizing antibodies factor) [13]. Recent data from a handful of patients who have undergone gene therapy for hemophilia B are very encouraging with a sustained factor IX level of $0.05 \mathrm{IU} / \mathrm{mL}$ maintained for over 4 years. Prophylaxis is also associated with high peak levels, which permits patients to maintain an active lifestyle [15].

Factor replacement therapy may be provided either "on demand" for symptoms related to bleeding or as "prophylaxis" in which scheduled infusions are undertaken in an attempt to prevent hemorrhage. Primary prophylaxis refers to factor replacement that is started to prevent clinical bleeding episodes in the infant or young child, while secondary prophylaxis refers to replacement therapy that is initiated in response to recurrent bleeding symptoms. Prophylaxis has the potential to change the landscape in hemophilia B by reducing debilitating musculoskeletal complications in patients with severe hemophilia and improving quality of life. Current clinical research and development efforts are predominantly aimed at manipulating the pharmacokinetic and physiologic properties of factor IX to prolong the biological half-life and/or enhance in vivo hemostatic function. Alternative approaches seek to "rebalance" the coagulation response via long-acting agents $[1,16]$.

Hemophilia is associated with an increased risk of blood transfusion after lower extremity total joint arthroplasty. Same care is needed to the femur surgery. Patients and providers should discuss these risks before surgery [17]. Before induction of anesthesia in a patient with hemophilia B should be performed one factor measurement, which is expected to be above $60 \%$ (preferably more than $80 \%$ ) for major surgery and $40 \%$ for minor procedures. In postoperative monitoring it's desirable that factor IX is between $30-50 \%$. In patients with hemophilia A the pre-operatory measurement must be preferably close to $100 \%$ (between $80-100 \%$ ) [13]. Hemophiliac patients' management has made strides. As a result of this development, it is imperative that new information, particularly identified with substitution treatment, be aced by hematologists as well as by the entire clinical-surgical group. The anesthesiologist ought to be responsible for receiving most satisfactory methodologies for hemophilia patients, effectively taking an interest and speaking with the individuals from a multidisciplinary group $[3,8,10]$.

\section{References}

1. Nazeef M, Sheehan JP (2016) New developments in the management of moderate-to-severe hemophilia B. J Blood Med 1: 27-38.

2. Boayue KB, Bell BA (1994) Von Willebrand disease. Int J Pediat Hem Onc 1: 449-61.

3. Flores RPG, Bagatini A, Santos ATL, Gomes CR, Fernandes MS, et al. (2004) Hemophilia and Anesthesia. Rev Bras Anestesiol 54: 865-71.

4. Acharya SS (2012) Exploration of the pathogenesis of haemophilic joint arthropathy: understanding implications for optimal clinical management. Br J Haematol 156: $13-23$.

5. White GC, Rosendaal F, Aledort LM, Lusher JM, Rothschild C, et al. (2001) Definitions in hemophilia. Recommendation of the scientific subcommittee on factor VIII and factor IX of the scientific and standardization committee of the International Society on Thrombosis and Haemostasis. Thromb Haemost 85: 560.

6. Lison S, Spannagl M (2014) Perioperative management of patients with hemophilia. Anaesthesist 63: 6-15.

7. Li Y, Weng XS, Lin J, Jin J, Qian WW, et al. (2016) Perioperative Period of a Hemophilia-related Osteoarthropathy Therapeutic Regimen and Analysis of Complications. Orthop Surg 8: 60-7.

8. Ferreira AA, Cangiani LM, Vanetti LFA (2004) Anestesia e o paciente hemofílico. Rev Bras Anestesiol 54: 467-74.

9. Makino S, Nomura Y, Kabara S, Takatsuji S, Kagawa T (2013) Anesthetic management of a patient with hemophilia B during scoliosis surgery. Masui 62: 1241-4. 10. Módolo NSP, Azevedo VLF, Santos PSS, Rosa ML, Corvino DR (2010) Anesthetic Strategy for Cesarean Section in a Patient with Factor XI Deficiency Case Report. Rev Bras Anestesiol 60: 176-80.

11. Shetty S, Ghosh K (2016) Why should hemophilia B be milder than hemophilia A? Haematologica 101: e213.

12. DiMichele D, Neufeld EJ (1998) Hemophilia. A new approach to an old disease. Hematol Oncol Clin North Am 12: 1315-44.

13. Caicedo MV, Raffan F, Duarte M (2009) Manejo perioperatorio del paciente hemofílico. Revista Mexicana de Anestesiología 32: 177-85.

14. World Federation of Hemophilia (2005) Guidelines for the Management of Hemophilia.

15. Giangrande P (2016) The Future of Hemophilia Treatment: Longer-Acting Factor Concentrates versus Gene Therapy. Semin Thromb Hemost 42: 513-7. 
16. Manco-Johnson MJ, Abshire TC, Shapiro AD, Riske B, Hacker MR, et al. (2007) Prophylaxis versus episodic treatment to prevent joint disease in boys with severe hemophilia. N Engl J Med 357: 535-44.

17. Kapadia BH, Boylan MR, Elmallah RK, Krebs VE, Paulino CB, et al. (2016) Does Hemophilia Increase the Risk of Postoperative Blood Transfusion After Lower Extremity Total Joint Arthroplasty? J Arthroplasty 31: 1578-82. 